\title{
Encrusted Suprapubic Catheter in Urethral Injury with Pulmonary Embolism: What To Do?
}

AA Hamzah ${ }^{1}$, AS Adnan ${ }^{2}$, TH Mallhi ${ }^{3 *}$, M Nor Gohar Rahman ${ }^{1}$, YH Khan ${ }^{3}$, AH Khan ${ }^{3}$

\begin{abstract}
Suprapubic catheters (SPC) have advantages over urethral catheters with respect to decreased bacteriuria and greater patient acceptability. Catheter encrustation is one of the most common intricacies of SPC and treated with extracorporeal shock wave lithotripsy and endoscopy lithotripsy. We reported a case of blocked, encrusted SPC along with urethral injury that was further complicated by pulmonary embolism. All conventional techniques to treat encrustation were not suitable due to worsening of urethral injury and risk of bleeding as patient was taking anti-coagulants. Therefore, we adopted a safe and quick procedure by which a $5 \mathrm{~mm} 0^{\circ}$ cystoscope was inserted and encrusted part of catheter was fragmented using artery forceps leading to removal of catheter without jeopardy to catheter track and patient`s safety.
\end{abstract}

Keywords: Catheter, pulmonary embolism, suprapubic catheter, urethral injury, urology From: ${ }^{1}$ Urology Unit, Department of Surgery, School of Medical Sciences, Universiti Sains Malaysia. ${ }^{2}$ Chronic Kidney Disease Resource Centre, School of Medical Sciences, Health Campus, University Sains Malaysia, Kubang Kerain 16150, Kelantan, Malaysia. ${ }^{3}$ Dicpline of Clinical Pharmacy, School of Pharmaceutical Sciences, University Sains Malaysia, Penang 11800, Malaysia

Correspondence: Dr TH Mallhi, Chronic Kidney Disease Resource Centre, School of Medical Sciences, Health Campus, University Sains Malaysia, Kubang Kerain 16150, Kelantan, Malaysia. Fax: +60978658956, e-mail: tauqeer.hussain.mallhi@hotmail.com

*AAH and MNGR performed procedure in current patient. ASA and AHK carried out radiological and laboratory investigations. THM and YHK wrote manuscript and approved final draft. 


\section{INTRODUCTION}

Urinary bladder catheters are used for urinary drainage or as a means to collect urine for measurement. Suprapubic catheters (SPC) are the most invasive catheter and require a surgical procedure for placement and insertion through the abdominal wall into the bladder either intraoperatively in association with another surgical procedure or percutaneously (1). Suprapubic catheterization has advantages over urethral catheterization with respect to decreased bacteriuria and greater patient acceptability (2). Avoidance of urethral strictures, decreased level of nursing care, preserved sexual function, prevention of penile pressure necrosis, early post-surgical ambulation, lower infection rates, ease of bladder training, shorter hospitalization stays and decreased postoperative infections are some other additional benefits of SPC (3). However SPC is also accompanied by several minor and rare major complications like bladder perforation, catheter migration, bleeding and blockage. Encrustation is one of the most common causes of catheter blockage that is usually treated with extracorporeal shock wave lithotripsy (ESWL) and endoscopy lithotripsy (EL). Here we reported a case of SPC encrustation with urethral injury complicated with pulmonary embolism for which patient was taking anticoagulants. We described a new technique for removal of SPC as patient was not suitable for conventional methods of SPC removal due to anticoagulant therapy and underlying urethral injury.

\section{CASE REPORT}

A 45-year-old Malay male alleged motor vehicle accident and had sustained open book pelvic fracture. Micturating cystouretrogram (MCUG) showed bulbous urethral injury (figure 1) for which suprapubic catheter was placed (Figure 2). Patient also had T10-L1 fracture with neurological deficit up to L1 level. Patient underwent open reduction and internal plate 
fixation for pelvic fracture and delayed repair was planned for urethral injury. Unfortunately, patient developed deep vein thrombosis (DVT) after surgery due to immobility for which low molecular weight heparin (LMWH) was initiated. Later, patient developed massive pulmonary embolism as a result of DVT and warfarin was started to maintain INR values within the range of 2-3. Patient was then discharged from hospital with SPC and was directed to change catheter after every 2 weeks.

One month later, patient was referred to urology outpatient clinic due to failure of catheter removal. An X-ray demonstrated catheter encrustation (Figure 3) while Computed Tomography (CT) scan of pelvic showed presence of calcification ring on the catheter suggesting encrustation.

All the conventional methods to treat encrustation were associated with high morbidity in this case due to use of anticoagulant therapy for pulmonary embolism. Repeated insertion of lithotripter through urethra was not suitable in our patient because of underlying urethral injury. So we adopted a quick and safe technique of removing encrusted SPC in this high risk patient. We admitted patient and planned procedure on $5^{\text {th }}$ day of admission. Warfarin was stopped and heparin was infused 3 days prior to procedure with monitoring of activated partial thromboplastin time (APTT) until its values were 2 times higher than normal. On procedure day, suprapubic tract was dilated by using artery forceps under spinal anaesthesia. Under the direct vision, a $5 \mathrm{~mm} 0^{\circ}$ cystoscope was inserted and encrusted part of catheter was fragmented using artery forceps leading to removal of catheter (Figure 4). Catheter track was thoroughly washed and a new SPC was placed. Oral warfarin was started immediately and patient was discharged on $2^{\text {nd }}$ day of procedure without any complication or bleeding episode. 


\section{Complicated Encrusted Suprapubic Catheter}

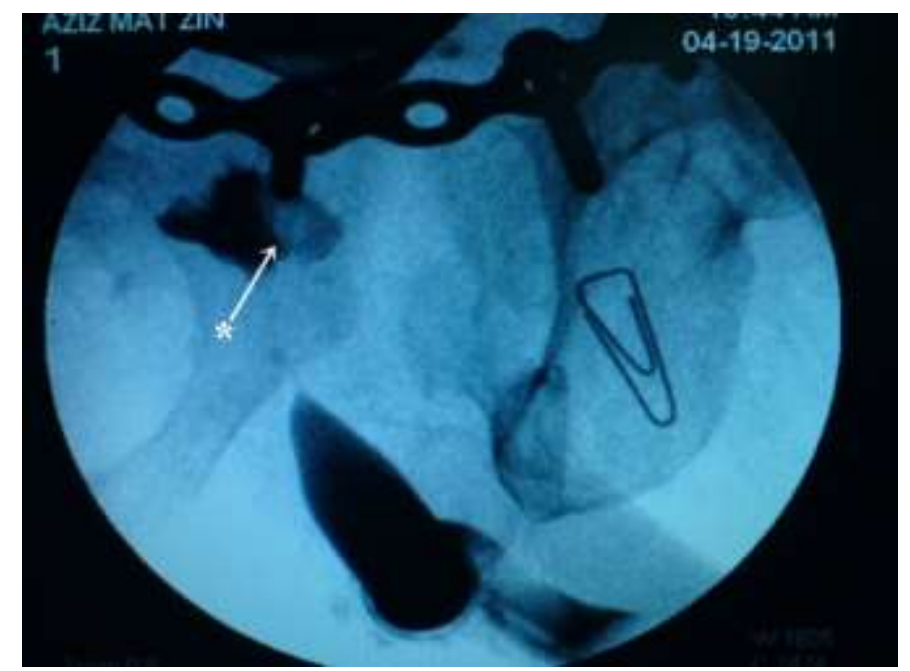

Fig 1: Micturating cystouretrogram (MCUG) indicating bulbous urethral injury $\left(^{*}\right)$

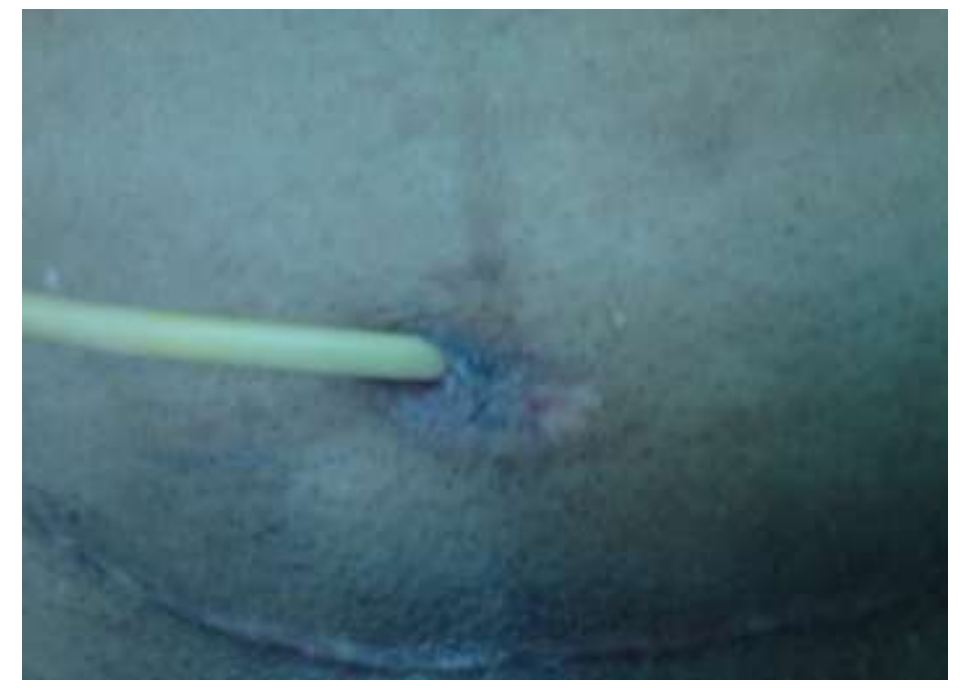

Fig 2 : Placement of suprapubic catheter due to delay repair for ureteral injury 


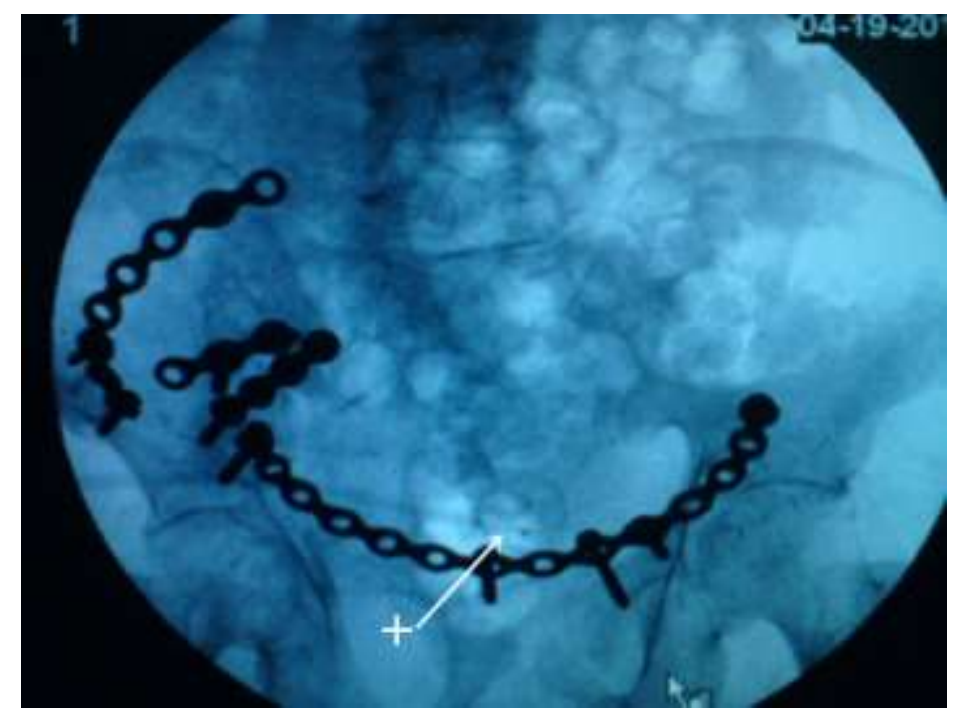

Fig 3 : X-Ray showing calcified ring suggesting encrusted catheter $(+)$
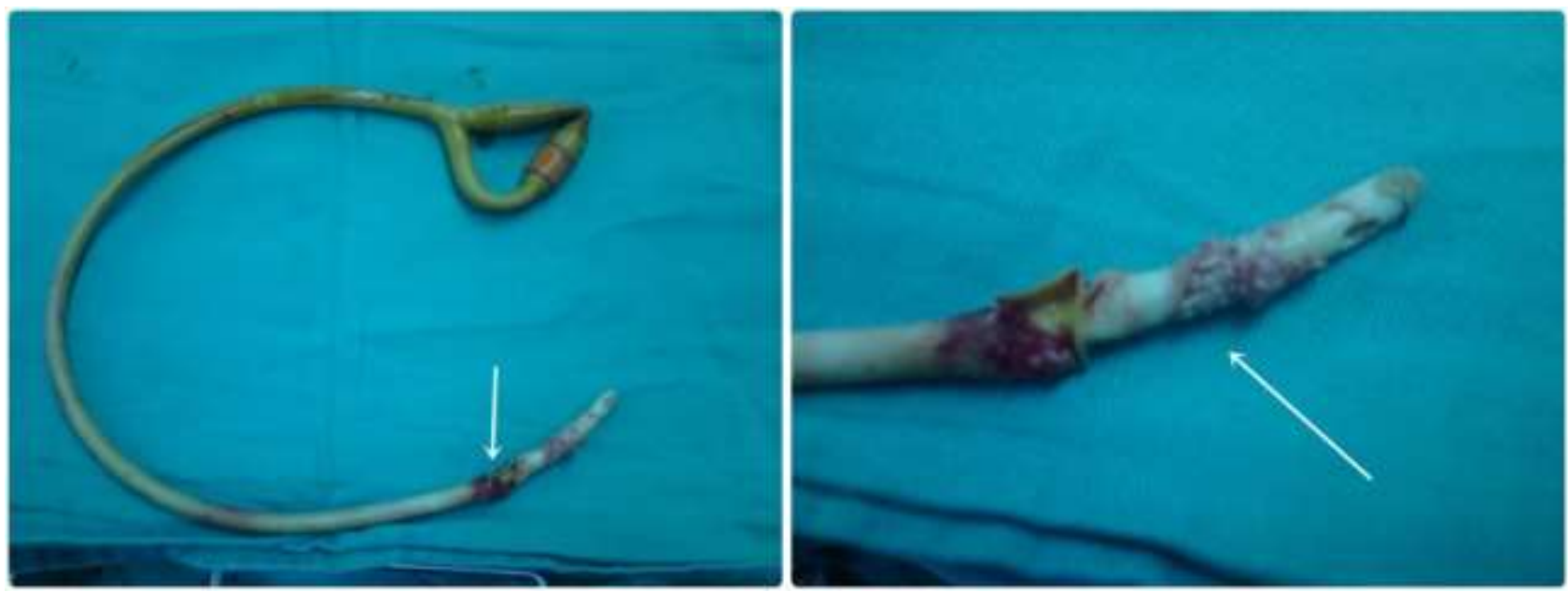

Fig 4: Removed suprapubic catheter with calcified tip

\section{DISCUSSION}

Urethral injury can be realigned by rigid cystoscopy in early phase of injury or by delayed urethral repair after 6 weeks of injury but in later case placement of SPC is needed to bypass 
the urethral (4). In our case, delayed repair was planned because patient had open book pelvic fracture with neurological deficit where prognosis is guarded. A long term catheterization was also planned due to high risk of neurogenic bladder. Catheter entrapment is a potential complication and occurs due to balloon malfunction, faulty valve mechanism, malfunction of the inflation channel or crystallization of fluid within the balloon (Mark 2003).

Besides of advantages of SPC over urethral catheterization it also carries several complications like infection, bladder spasm, balloon malfunction and intraluminal or extraluminal catheter encrustation (2). The encrustation mechanism can be explained by the reason that insertion of catheter in body causes immune response leading to formation of bacterial biofilm on catheter. This biofilm secretes an extracellular matrix of bacterial glycocalyces and host proteins. Urinary crystals such as struvite and calcium phosphate are also incorporated into biofilm (6). Alkalization of urine by urease enzyme, released by Proteus mirabilis, promotes crystallization of struvite and calcium phosphate (7).

Under normal circumstances encrustation of catheter can be treated either with ESWL or EL. Canby-Hagino et al described that use of intraluminal pneumatic lithotripsy for removal of intraluminal encrustation of the catheter; a pneumatic lithotripsy probe is inserted into the lumen of the catheter and advanced in a jackhammer-like fashion. This technique results in disruption of intraluminal encrustations and straightening of the tubes so that they can be removed in an atraumatic manner (8). Kunzman et al reported that use of ESWL delivered at $8 \mathrm{kV}$ level causes fragmentation of encrusted balloon (9). Christopher Ho reported a case in which encrusted urethral catheter was fragmented by using lithotriptor under direct vision through suprapubic catheter tract (10).

We faced management dilemma in current case. Placement of urethral catheter was contraindicated due to blocked, encrusted SPC and underlying urethral injury. Treatment of encrustation with ESWL was not suitable because of warfarin use and possible risk of 
bleeding during procedure. Use of lithotripter requires repeated insertion through urethral that can worsen the urethral injury. Therefore, we adopted a safe and quick procedure to remove encrusted SPC that did not jeopardize the catheter track and patient's safety.

\section{CONCLUSION}

Encrusted catheter is a common intricacy in urology practice that can be treated by several ways. To our best knowledge, we reported first case of treatment of encrustation with artery forceps by using $5 \mathrm{~mm} 0^{\circ}$ cystoscope in high risk patient with pulmonary embolism and urethral injury where conventional methods of treatment are contraindicated.

\section{Learning points}

Current report is a rare case with suprapubic catheter (SPC) encrustation complicated with urethral injury and pulmonary embolism

Extracorporeal shock wave lithotripsy (ESWL) and endoscopy lithotripsy are conventional and gold standards to manage such patients in routine practice

Conventional treatments for encrustation were not suitable in our patient because of urethral injury and anticoagulants use.

We developed a safe and quick technique to break encrusted SPC without harming catheter track.

A new technique used to treat such high risk patient will aid clinicians to manage such patients in safely manners. 


\section{REFERENCES}

1. Jacob P, Rai BP, Todd AW. Suprapubic catheter insertion using an ultrasound-guided technique and literature review. BJU Int 2012; 110: 779-84.

2. Perrin LC, Penfold C, McLeish A. A prospective randomized controlled trial comparing suprapubic with urethral catheterization in rectal surgery. Aust N Z J Surg 1997; 67: 554-6.

3. Adeyemo B, Makovitch S, Foo D. A peculiar complication of suprapubic catheterization: Recurrent ureteral obstruction and hydronephrosis. J Spinal Cord M 2013; 36: 166-9.

4. Olapade-Olaopa EO, Adebayo SA, Atalabi OM, Popoola AA, Ogunmodede IA, Enabulele UF. Rigid retrograde endoscopy under regional anaesthesia: a novel technique for the early realignment of traumatic posterior urethral disruption. Afr $\mathrm{J}$ Med Med Sci 2002; 31: 227-80

5. Gonzalgo ML, Patrick CW. Balloon cuffing and management of the entrapped foley catheter. Urology 2003; 61: 825-7.

6. Hedelin H, Eddeland A, Larsson L, Pettersson S, Ohman S. The composition of catheter encrustations, including the effects of allopurinol treatment. Br J Urol 1984; 56: $250-54$.

7. Mobley HL, Warren JW. Urease-positive bacteriuria and obstruction of long-term urinary catheters. J Clin Microbiol 1987; 25: 2216-17.

8. Canby-Hagino ED, Caballero RD, Harmon WJ. Intraluminal pneumatic lithotripsy for the removal of encrusted urinary catheters. J Urol 1999; 162: 2058-60.

9. Kunzman SA, Srinadh ES, Lala SM, Albusaidi Q. Management of retained encrusted urethral catheter with extracorporeal shockwave lithotripsy. Indian J Urol 2002; 19: $83-4$. 
10. Christopher CK Ho, Yugasaravanan Khandasamy, Praveen Singam, Eng Hong Goh and Zulkifli Zainuddin. Encrusted and incarcerated urinary bladder catheter: what are the options? Libyan J Med 2010; 5. 